\title{
RETRACTED ARTICLE: Summer Research Training Provides Effective Tools for Underrepresented Minorities to Obtain Doctoral Level Degrees
}

\author{
Oluwatoyin A. Asojo $^{1}$ - Ashish Damania ${ }^{1}$ - Teri L. Turner ${ }^{2}$ Gayle Slaughter $^{3}$. \\ Kendal D. Hirschi ${ }^{4}$
}

Received: 20 November 2016 /Revised: 17 December 2016 / Accepted: 19 December 2016/Published online: 11 January 2017

(C) The Author(s) 2017. This article is published with open access at Springerlink.com

The editor-in-chief has recommended retraction due to irregularities with the reporting of the primary data set. The online version of this article contains the full text of the retracted article as electronic supplementary material.

The editor-in-chief has recommended retraction due to irregularities with the reporting of the primary data set. The online version of this article contains the full text of the retracted article as electronic supplementary material.

Electronic supplementary material The online version of this article (https://doi.org/10.1007/s40615-016-0330-0) contains supplementary material, which is available to authorized users.

\section{Oluwatoyin A. Asojo}

asojo@bcm.edu

1 National School of Tropical Medicine, Baylor College of Medicine, Houston, TX 77030, USA

2 Center for Research, Innovation and Scholarship in Medical Education, Texas Children's Hospital, Department of Pediatrics, Baylor College of Medicine, Houston 77030, TX, USA

3 Graduate School, Baylor College of Medicine, Houston 77030, TX, USA

4 Children's Nutritional Research Center, Baylor College of Medicine, Houston 77030, TX, USA 